\title{
Frequency-Selective Adaptive Control of a Hybrid Suspension System *
}

\author{
Nils Pletschen* Sebastian Spirk* Boris Lohmann * \\ * Institute of Automatic Control, Technische Universität München, \\ 85748 Garching, Germany (e-mail: nils.pletschen@tum.de).
}

\begin{abstract}
This paper deals with a frequency-selective reference model that is used within an adaptive control structure for mechatronic vehicle suspensions. Based on a hybrid actuator configuration which consists of a slow-active actuator and a semi-active damper, the basic idea of the control approach is to emulate the dynamic behavior of a passive, but time-variant reference suspension being optimally attuned for the current driving state. The new reference incorporates the limited bandwidth of the active device in the design step and is also able to address the divergent damping demand of sprung and unsprung mass of modern vehicles. An additional potential of the resulting frequency-selective reference model of up to $25 \%$ in comfort is revealed in comparison to the original reference without frequency-dependent properties. However, as suggested by a comparative study in terms of simulation results of a quarter-car setting based on real series suspension components this potential depends heavily on the restrictions that are imposed by the available damper characteristics.
\end{abstract}

Keywords: Active suspensions; Semi-active dampers; Vehicle dynamics; Frequency-dependent characteristics; Adaptive control.

\section{INTRODUCTION}

Despite the fact that vehicle suspensions have been subject to continuous development, the conflicting tasks of providing excellent ride comfort and ride safety, while simultaneously meeting constructional constraints, has not yet been solved satisfactorily. While ride comfort asks for an isolation of the vehicle body from the road unevenness and thus a soft passive suspension configuration, ride safety is reached by a rather firm spring and damper setting to ensure good tire-road contact for the transfer of steering and braking forces. Meanwhile, the use of active and semi-active suspension configurations can ease the aforementioned conflict, Hrovat (1997). Although fully active actuators offer the largest performance potential, semi-active components have mainly prevailed within production vehicles due to their lower power consumption and manufacturing costs. The combination of a slow-active actuator with a bandwidth of up to $5 \mathrm{~Hz}$ in series to a passive spring and a semi-active continuously variable damper, see Fig. 1 (mid.), is able to combine the advantages of both components. As has been shown in Koch et al. (2010a), the performance potential of this so-called hybrid suspension comes close to that of a fully active suspension configuration at a considerably lower power demand.

The potential of the respective actuator configurations can best be exploited when an adaptive control law is applied, i. e. when there is not a fixed trade-off between ride comfort and ride safety, but rather a shift in controller parameterization according to the current vehicle states and road characteristics. This idea has been taken on in Koch et al. (2010b), where the hybrid actuator combination is used to emulate the dynamic behavior of a passive reference model

\footnotetext{
^ This project at the Institute of Automatic Control, TU München has been funded by the German Research Foundation (DFG).
}

with time-variant stiffness and damping that is optimally tuned for the current driving state, Fig. 1 (ri.).

Based on the main idea of the aforementioned adaptive control concept, where it is assumed that a desired spring stiffness can be emulated by the low-bandwidth active actuator across the entire relevant frequency range, the aim of this paper is to take into account the actual available hardware configuration already for the design of the reference model. Also, due to the difference in mass and related stiffness, the damping demand of sprung and unsprung mass differs. Therefore, a frequency-selective reference model is proposed that is able to realize different stiffness and damping values for the low-frequent and highfrequent motions in the relevant frequency range.

\section{SUSPENSION CONCEPT}

\subsection{Suspension Model}

In the frequency range below $25 \mathrm{~Hz}$, the major dynamic effects relevant for the analysis of the vertical motion of vehicle body and wheel mass can be studied by means of quarter-car models, Mitschke and Wallentowitz (2004). Based on the quarter-car model of the hybrid suspension configuration in Fig. 1 (mid.), the equations of motion for the chassis mass $m_{\mathrm{c}} \approx 500 \mathrm{~kg}$ and the wheel mass $m_{\mathrm{w}} \approx 70 \mathrm{~kg}$ are derived by applying Newton's law. Together with the state vector $\boldsymbol{x}(t)=\left[z_{\mathrm{c}}-z_{\mathrm{w}}, \dot{z}_{\mathrm{c}}, z_{\mathrm{w}}-z_{\mathrm{g}}, \dot{z}_{\mathrm{w}}\right]^{T}$, the input
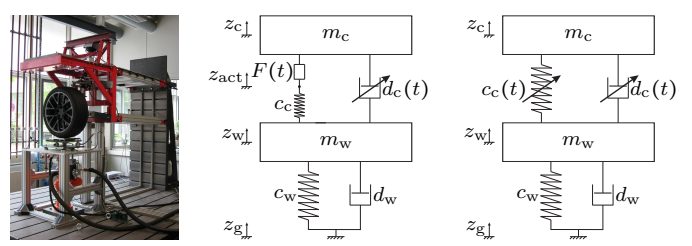

Fig. 1. Test rig (le.) and model of the hybrid quarter-car (mid.) and time-variant passive reference model (ri.) 
vector $\boldsymbol{u}(t)=\left[u_{\mathrm{hy}}, u_{\mathrm{cvd}}, u_{\mathrm{d}}\right]^{T}$ and the output vector $\boldsymbol{y}(t)=$ $\left[\ddot{z}_{\mathrm{c}}, F_{\mathrm{dyn}}, z_{\mathrm{cw}}\right]^{T}$ these can be transformed into a fourthorder state space model. Therein, $\boldsymbol{y}$ contains the chassis acceleration $\ddot{z}_{\mathrm{c}}$, the dynamic tire load $F_{\mathrm{dyn}}=-c_{\mathrm{w}}\left(z_{\mathrm{w}}-z_{\mathrm{g}}\right)$ and the suspension travel $z_{\mathrm{cw}}=z_{\mathrm{c}}-z_{\mathrm{w}}$, and hence all of the quantities that are relevant for performance evaluation, see Section 2.2. While the deflection of the hydraulic spring mount adjustment $u_{\text {hy }}(t)=z_{\mathrm{c}}(t)-z_{\text {act }}(t)$ and the semiactive damper force $u_{\text {cvd }}(t)=F_{\mathrm{d}}(t)$ serve as control inputs, $u_{\mathrm{d}}(t)=\dot{z}_{\mathrm{g}}(t)$ symbolizes the road-induced disturbance.

On the basis of the suspension components, the kinematics, the actuators and the sensor architecture of a current upperclass sedan, a quarter-car test rig has been designed for experimental analysis of suspension control concepts, see Fig. 1 (le.). For accurate simulation results and control input calculation, the major nonlinear effects such as a progressive spring characteristic, degressive force-velocity characteristics of the semi-active damper with an asymmetric behavior in the compression and tension direction and the frequency-dependent characteristics of the tire have been identified at the test rig and are taken into account, see Koch et al. (2011b). In order to complete the realistic setup, the measurement signals used for suspension control are $\boldsymbol{y}_{\mathrm{m}}=\left[\ddot{z}_{\mathrm{c}}, \ddot{z}_{\mathrm{w}}, z_{\mathrm{cw}}\right]^{T}$, which resembles a common sensor configuration for modern production vehicles. Other quantities needed for control purposes, such as the damper relative velocity $\hat{\dot{z}}_{\mathrm{cw}}$ and the dynamic tire load $\hat{F}_{\text {dyn }}$, are estimated by means of a signal-based filtering approach, see Koch et al. (2010b). The passive suspension configuration is realized by keeping the spring mount adjustment at a constant level and applying a constant current to the damper valves such that a medium damper characteristic results. Hence,

$$
f_{\mathrm{c}, \mathrm{p}}=\frac{1}{2 \pi} \sqrt{\frac{c_{\mathrm{c}}}{m_{\mathrm{c}}}} \approx 1.1 \mathrm{~Hz} \quad \text { and } \quad D_{\mathrm{c}, \mathrm{p}}=\frac{d_{\mathrm{c}}}{2 \sqrt{c_{\mathrm{c}} m_{\mathrm{c}}}} \approx 0.21
$$

are attained as undamped natural frequency and damping ratio of the body mass, respectively.

\subsection{System specification}

The system requirements have been derived based on the real suspension, see Koch et al. (2011b):

- Ride comfort: Especially in the comfort-relevant frequency range between 4 to $8 \mathrm{~Hz}$, the human body is most sensitive to vertical vibration. Thus, ride comfort is quantified by the root mean square (rms) value $\left\|\ddot{z}_{\mathrm{c}, \text { iso }}(t)\right\|_{\mathrm{rms}}$ of the weighted chassis acceleration $\ddot{z}_{\mathrm{c} \text {, iso }}(t)$, which is calculated by applying the shaping filter given in ISO (1997) to the chassis acceleration signal $\ddot{z}_{\mathrm{c}}(t)$. The lower the rms and peak values of the acceleration, the higher the ride comfort.

- Ride safety: For good tire-road contact, the dynamic tire load $F_{\text {dyn }}$ should be minimized. In order to prevent wheel lift-off, $F_{\text {dyn }}$ should not exceed the negative value of the static tire load $F_{\text {stat }}$. When a Gaussian distribution of the dynamic tire load is assumed, a constraint for its rms value

$$
\left\|F_{\text {dyn }}\right\|_{\mathrm{rms}} \leq \frac{1}{3}\left(m_{\mathrm{c}}+m_{\mathrm{w}}\right) \cdot g=\frac{F_{\text {stat }}}{3}
$$

can be defined, Mitschke and Wallentowitz (2004).

- Suspension deflection limits: Constructional suspension travel limitations $\underline{z}_{\mathrm{cw}} \leq z_{\mathrm{cw}}(t) \leq \bar{z}_{\mathrm{cw}}$ must be met.

- Actuator power demand: The power consumption of the spring mount adjustment should be minimized. It is quantified by the average of the positive mechanical actuator power as defined in Koch et al. (2011b)

$$
\begin{aligned}
\overline{P^{+}} & =\frac{1}{T} \int_{0}^{T} P^{+}(\tau) \mathrm{d} \tau, \\
P^{+}(t) & = \begin{cases}F(t) \Delta \dot{z}_{\text {act }}(t) & \text { for } F(t) \Delta \dot{z}_{\text {act }}>0 \\
0 & \text { else. }\end{cases}
\end{aligned}
$$

\section{FREQUENCY-SELECTIVE CONTROL CONCEPT}

The controller concept examined in this paper is based upon the adaptive control approach proposed in Koch et al. (2010b) and experimentally validated in Koch et al. (2011b). The idea of the original concept is to determine the desired suspension forces by means of a passive suspension setting (natural frequency $f_{\mathrm{c}}$ and damping ratio $D_{\mathrm{c}}$ of the sprung mass) that is most suited for the current driving state. Therefore, the criticality of the current driving condition with respect to the dynamic tire load $F_{\text {dyn }}$ and the suspension deflection $z_{\mathrm{cw}}$ is monitored such that two scheduling parameters $0 \leq q_{\mathrm{fdyn}}, q_{\mathrm{susp}} \leq 2$ are determined. Adaptation signals close to zero, i. e. when the vehicle state is uncritical, enable a very comfortable passive suspension setting with a low chassis eigenfrequency and a low chassis damping ratio. When the driving conditions become rougher and thus $q_{\mathrm{fdyn}}$ and/or $q_{\text {susp }}$ increase, damping and stiffness of the passive reference are raised according to the Pareto-optimal front within the conflict diagram relating comfort-weighted acceleration and dynamic tire load. As a result, stiffness and damping parameters are chosen such that the highest possible comfort is achieved without violating predetermined limits in terms of ride safety. By means of this adaptive control approach, which is based on a passive reference model with time-varying stiffness and damping, the desired forces $F_{\mathrm{cw}}(t)=F_{\mathrm{s}}(t)+F_{\mathrm{d}}(t)$ between sprung and unsprung mass are determined for each instant of time. Subordinate actuator controllers are then responsible for tracking of these commanded forces, see Koch et al. (2011a).

\subsection{Motivation for frequency selection}

Given that the optimal passive suspension setting in terms of $f_{\mathrm{c}}$ and $D_{\mathrm{c}}$ is determined by assuming idealized timevarying stiffness and damping, its feasibility by means of the available actuators is not ensured. In fact, the desired eigenfrequency can only be emulated by the spring mount adjustment within the low-frequency range of up to $5 \mathrm{~Hz}$. As illustrated in Fig. 2, spring deflections resulting from relative motion of body and wheel mass that lie above the actuator bandwidth cannot be altered; thus spring forces are realized according to the invariant physical stiffness $c_{\mathrm{c}, \text { phys }}$ of the coil spring. Moreover, since the natural frequencies of sprung and unsprung mass lie in different regions of the relevant frequeny range, due to the large difference in their respective masses and related stiffnesses, the damping demand of body and wheel differ. While it might be advantageous for vehicle body isolation to provide less resistance to high-frequency motions of the comparatively small wheel mass without losing in roadholding performance, higher damping values are needed to provide sufficient attenuation of the low-frequent movement of the considerable larger chassis mass $\left(\frac{m_{\mathrm{c}}}{m_{\mathrm{w}}} \approx 7\right)$. This issue has been addressed before through constructionally amplitude-selective or frequency-selective damping devices, e.g. Boivin (2010), de Kock and de Ruiter 


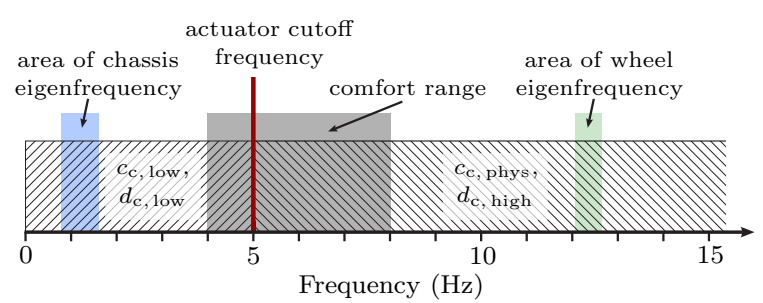

Fig. 2. Frequency-selective stiffness and damping ranges

(2008). Thus, in order to give some more flexibility to the reference model, also frequency-dependent damping with $d_{\mathrm{c} \text {, low }}$ and $d_{\mathrm{c} \text {, high }}$ is introduced in the low-frequency and the high-frequency range, respectively, see Fig. 2.

\subsection{Filtering}

In order to realize a time-variant passive reference model with frequency-selective stiffness and damping characteristics, the relevant time signals have to be separated with respect to the frequency spectrum included. This task can be performed by applying lowpass and highpass filters to the suspension deflection $z_{\mathrm{cw}}$ and the damper relative velocity $\dot{z}_{\mathrm{cw}}$. However, when done in a real-time application, filtering inevitably leads to phase shifts, which may distort the resulting time signals. Besides the attenuation in magnitude above the cutoff frequency $f_{\mathrm{lp}}$ a first-order Butterworth lowpass filter of the form

$$
H_{\mathrm{lp}}(s)=\frac{1}{1+T_{\mathrm{lp}} s},
$$

where $s$ and $T_{\mathrm{lp}_{\mathrm{p}}}=\frac{1}{2 \pi f_{\mathrm{lp}}}$ denote the Laplace variable and the time constant of the filter, respectively, time signals experience a phase lag of -45 degrees even at $f_{\mathrm{lp}}$, which increases to a maximum phase lag of up to -90 degrees for higher frequencies. Similarly, a first-order Butterworth highpass filter with the transfer function

$$
H_{\mathrm{hp}}(s)=\frac{T_{\mathrm{hp}} s}{1+T_{\mathrm{hp}} s}
$$

is able to attenuate frequencies up to its cutoff frequency $f_{\mathrm{hp}}$, where time signals pass through the filter with a phase lead of up to +90 degrees for frequencies $f \rightarrow 0$. However, by choosing the same time constant $T_{\mathrm{lp}} \equiv T_{\mathrm{hp}}$ for the lowpass and the highpass filter, respectively, when both filtered time signals are summed up, the distortions cancel each other out, i. e.

$$
H_{\mathrm{lp}+\mathrm{hp}}(s)=H_{\mathrm{lp}}(s)+H_{\mathrm{hp}}(s)=1 .
$$

The combined signal shows neither a change in magnitude nor a phase shift. While a sharper separation of frequencies is enabled by a higher filtering order, this also leads to increased phase shifts and the effect of canceled-out distortions does no longer hold. Thus, as is illustrated in Fig. 3, a combination of first-order lowpass and highpass filters, both featuring the same time constant $T_{\mathrm{lp}}=T_{\mathrm{hp}}$, is chosen

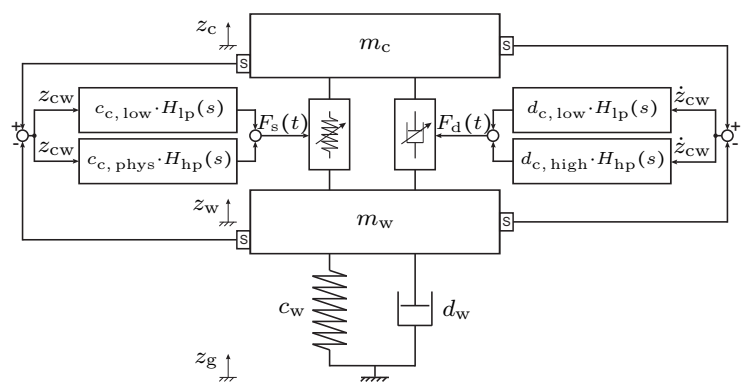

Fig. 3. Frequency-selective passive reference model for the frequency-selective reference model. Although in this case the sums of the lowpass- and highpass-filtered suspension deflection or damper relative velocity signal resemble the unfiltered quantity, respectively, obviously the summation of spring and damper forces calculated from the filtered signals using different spring and damping constants $c_{\mathrm{c} \text {, low }} \neq c_{\mathrm{c}}$, phys and $d_{\mathrm{c} \text {, low }} \neq d_{\mathrm{c}}$, high do not equal the forces determined from the unfiltered signals when applying one global parameter, respectively.

\subsection{Frequency-selective reference models}

As in Koch et al. (2011b), conflict diagrams are used to determine the optimal configurations of the chassis natural frequency and the chassis damping ratio. These diagrams are created offline by means of simulations of the passive reference model, which is parametrized by various combinations of $f_{\mathrm{c}}$ and $D_{\mathrm{c}}$. In contrast to the reference modelbased control without frequency selection, the number of free parameters is increased: Not only do one global natural frequency $f_{\mathrm{c}}$ and one global damping ratio $D_{\mathrm{c}}$ vary, but rather one eigenfrequency $f_{\mathrm{c} \text {, low }}$ (referring to a stiffness $\left.c_{\mathrm{c}, \text { low }}\right)$ for the low-frequency range and two damping ratios $D_{\text {c, low }}$ and $D_{\mathrm{c}, \text { high }}$ (referring to damping coefficients $d_{\mathrm{c} \text {, low }}$ and $\left.d_{\mathrm{c} \text {, high }}\right)$ for the low-frequency and the high-frequency range, respectively, are defined as free variables. Therefore, lowpass and highpass filters, as discussed in Section 3.2, are introduced into a simplified version of the quarter-car model, where some nonlinearities and actuator dynamics have been neglected. Instead, a linear primary spring and an asymmetric damper characteristic linearized separately for the compression and the tension direction are assumed. The overall damping ratio is hence computed from the mean value of both linear damping coefficients. Two different versions of calculating frequency-selective spring and damper forces are analyzed:

Type 1 The first alternative makes use of the combination of lowpass and highpass filters, where the filter time constants are identical, as discussed in the previous section. So for a fixed parameter set $\left\{f_{\mathrm{c}, \text { low }}, D_{\mathrm{c}, \text { low }}, D_{\mathrm{c}, \text { high }}\right\}$ and thus for $\left\{c_{\mathrm{c} \text {, low }}, d_{\mathrm{c} \text {, low }}, d_{\mathrm{c}, \text { high }}\right\}$, the spring and damping forces are calculated by

$$
\begin{aligned}
& F_{\mathrm{s}, 1}(t)=-c_{\mathrm{c}, \text { low }} \cdot z_{\mathrm{cw}, \text { low }}(t)-c_{\mathrm{c}, \text { phys }} \cdot z_{\mathrm{cw}, \text { high }}(t), \\
& F_{\mathrm{d}, 1}(t)=-d_{\mathrm{c}, \text { low }} \cdot \dot{z}_{\mathrm{cw}, \text { low }}(t)-d_{\mathrm{c}, \text { high }} \cdot \dot{z}_{\mathrm{cw}, \text { high }}(t),
\end{aligned}
$$

respectively. The low-frequent and high-frequent suspension deflection, as well as the low-frequent and highfrequent damper relative velocity, are thereby indicated by $z_{\mathrm{cw}, \text { low } / \text { high }}(t)$ and $\dot{z}_{\mathrm{cw} \text {, low } / \text { high }}(t)$ such that

$$
\begin{aligned}
\mathcal{L}\left\{z_{\mathrm{cw}, \text { low }}(t)\right\} & =Z_{\mathrm{cw}, \text { low }}(s)=H_{\mathrm{lp}}(s) \cdot Z_{\mathrm{cw}}(s), \\
\mathcal{L}\left\{z_{\mathrm{cw}, \operatorname{high}}(t)\right\} & =Z_{\mathrm{cw}, \operatorname{high}}(s)=H_{\mathrm{hp}}(s) \cdot Z_{\mathrm{cw}}(s)
\end{aligned}
$$

with $H_{\mathrm{lp}}(s)$ and $H_{\mathrm{hp}}(s)$ from (4) and (5) hold.

Type 2 The second case is based on the consideration that though the sum of first-order lowpass and highpass filters does not introduce a phase shift to the signal, the individual quantities themselves are indeed distorted. Thus the spring and damping forces generated from filtered signals might not be applied at the correct instant of time, but might rather engage too early or too late. This is especially critical for the realization of damping, since damping dissipates energy from the system.

In order to respond to this issue, the second type of frequency-selective reference model features a basic stiff- 


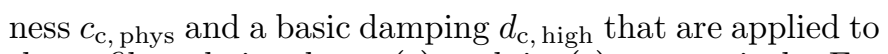
the unfiltered signals $z_{\mathrm{cw}}(t)$ and $\dot{z}_{\mathrm{cw}}(t)$, respectively. For the realization of frequency-selective reference forces, the stiffness difference $\Delta \underline{c}_{\mathrm{c}}=c_{\mathrm{c}, \text { low }}-c_{\mathrm{c} \text {, phys }}$ and the damping difference $\Delta \underline{d}_{\mathrm{c}}=d_{\mathrm{c}, \text { low }}-d_{\mathrm{c}}$, high, which are to be additionally effective for the low-frequency motions $z_{\mathrm{cw}}$, low $(t)$ and $\dot{z}_{\mathrm{cw}}$, low $(t)$ are then added to these basic forces. Thus the overall spring and damping forces are calculated by

$$
\begin{aligned}
& F_{\mathrm{s}, 2}(t)=-c_{\mathrm{c}, \text { phys }} \cdot z_{\mathrm{cw}}(t)-k_{1} \cdot \Delta \underline{c}_{\mathrm{c}} \cdot z_{\mathrm{cw}, \text { low }}(t), \\
& F_{\mathrm{d}, 2}(t)=-d_{\mathrm{c}, \text { high }} \cdot \dot{z}_{\mathrm{cw}}(t)-k_{2} \cdot \Delta \underline{d}_{\mathrm{c}} \cdot \dot{z}_{\mathrm{cw}, \text { low }}(t),
\end{aligned}
$$

where $k_{1}$ and $k_{2}$ are scaling factors defined as follows. Calculating the total force $F_{\mathrm{cw}, 2}(t)=F_{\mathrm{s}, 2}(t)+F_{\mathrm{d}, 2}(t)$ between body and wheel mass in the Laplace domain yields

$$
\begin{aligned}
F_{\mathrm{cw}, 2}(s)= & -\left[c_{\mathrm{c}, \text { phys }}+k_{1} \Delta \underline{c}_{\mathrm{c}} H_{\mathrm{lp}}(s)\right. \\
& \left.+s d_{\mathrm{c}, \text { high }}+s k_{2} \Delta \underline{d}_{\mathrm{c}} H_{\mathrm{lp}}(s)\right] \cdot Z_{\mathrm{cw}}(s)
\end{aligned}
$$

and with $s=j \omega$, after some conversions, finally results in

$$
\begin{gathered}
F_{\mathrm{cw}, 2}(j \omega)=(\underbrace{-\frac{\left(1+T_{\mathrm{lp}}^{2} \omega^{2}\right) c_{\mathrm{c}, \mathrm{phys}}+k_{1} \Delta_{\underline{c}_{\mathrm{c}}}+k_{2} T_{\mathrm{lp}} \omega^{2} \Delta \underline{d}_{\mathrm{c}}}{1+T_{\mathrm{lp}}^{2} \omega^{2}}}_{\operatorname{Re}(\star)} \\
-j \underbrace{\omega \frac{\left(1+T_{\mathrm{lp}}^{2} \omega^{2}\right) d_{\mathrm{c}, \mathrm{high}}+k_{2} \Delta \underline{d}_{\mathrm{c}}-k_{1} T_{\mathrm{lp}} \Delta \underline{c}_{\mathrm{c}}}{1+T_{\mathrm{lp}}^{2} \omega^{2}}}_{-\operatorname{Im}(\star)}) \cdot Z_{\mathrm{cw}}(j \omega) .
\end{gathered}
$$

As stiffness in this context is characterized by the real part and damping is denoted by the imaginary part of the bracket term $\star(j \omega)$ in $(14)$, it can be noted that filtering of the signals causes an interference in the spring and damping forces: Stiffness is no longer realized only by the spring, but is also introduced by phase shifted damping. A similar statement is valid for the actually realized damping force. However, in order to obtain spring and damping forces as desired at least for a particular frequency of interest $\bar{\omega}<\frac{1}{T_{\mathrm{p}}}$ within the low-frequency range,

$$
-\operatorname{Re}(\star(j \bar{\omega})) \stackrel{!}{=} c_{\mathrm{c}, \text { low }} \quad \text { and } \quad-\operatorname{Im}(\star(j \bar{\omega})) \stackrel{!}{=} \bar{\omega} d_{\mathrm{c}, \text { low }}
$$

must hold. Solving this system of equations with respect to $k_{1}$ and $k_{2}$ results in the scaling factors

$$
k_{1}=1-T_{\mathrm{lp}} \bar{\omega}^{2} \frac{\Delta \underline{d}_{c}}{\Delta \underline{c}_{\mathrm{c}}} \quad \text { and } \quad k_{2}=1+T_{\mathrm{lp}} \frac{\Delta \underline{\underline{c}}_{\mathrm{c}}}{\Delta \underline{\underline{d}}_{\mathrm{c}}} .
$$

Since the bandwidth of the spring mount adjustment is limited to $5 \mathrm{~Hz}$ and for the reasons named before, in both the reference model alternatives the filter time constants are set to $T_{\mathrm{lp}}=T_{\mathrm{hp}}=\frac{1}{2 \pi \cdot 5 \mathrm{~Hz}} \approx 0.032 \mathrm{sec}$. For the reference model of Type 2 , the relevant frequency range is around the chassis eigenfrequency; hence for calculating the scaling factors, a medium value $\bar{\omega}=2 \pi f_{\mathrm{c}, \mathrm{p}}$ is chosen according to the chassis natural frequency of the passive suspension configuration, see Section 2.1.

\subsection{Conflict diagram and Pareto-optimal front}

On the basis of the reference models introduced above, conflict diagrams are generated where each point in the socalled carpet plot results from a nonlinear simulation of the quarter-car model traveling over a stochastic road profile. Since the road height velocity $\dot{z}_{\mathrm{g}}$ is the disturbance input to the model, white noise is used as excitation, see Mitschke and Wallentowitz (2004). The road parameters are chosen such that the excitation corresponds to a highway of medium quality, which is traveled at a vehicle speed of $v_{\text {veh }}=180 \frac{\mathrm{km}}{\mathrm{h}}$, but simultaneously resembles a bad country road traveled at a lower velocity.
The upper part of Fig. 4 depicts the resulting conflict diagram with respect to weighted chassis acceleration and dynamic tire load when the frequency-selective reference model of Type 1 is applied. The influence of the highfrequency damping ratio can be observed on the $y$-axis. Thus each line in the diagram denotes a parameter set of fixed natural frequency $f_{\mathrm{c} \text {, low }}$ and fixed damping ra-

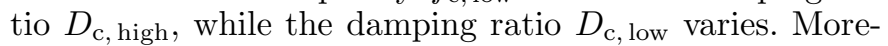
over, the comfort-optimal high-frequency damping ratio (COHD) and the safety-optimal high-frequency damping ratio (SOHD) are marked in Fig. 4 (upper). In the lower part of Fig. 4, the Pareto-optimal front for Type 1 of the frequency-selective reference model derived from the carpet plot above is illustrated by the red dashed line. For reasons of comparison, the green solid line shows the Pareto-optimal front that results from the standard reference model without frequency selection. The values of chassis eigenfrequency and chassis damping ratio are restricted to a reasonable variation range. In both cases, the natural frequency is allowed to vary between $0.8 \mathrm{~Hz} \leq f_{\mathrm{c}}, f_{\mathrm{c} \text {, low }} \leq 1.6 \mathrm{~Hz}$. Due to considerations of lowfrequent roll motion and due to the fact that no damping would lead to an excessive increase in amplitude resulting from forced vibration particularly within the resonance frequency range of the chassis mass the damping ratio is bounded by $0.18 \leq D_{\mathrm{c}}, D_{\mathrm{c} \text {, low }} \leq 0.7$. In the high-frequency band around the wheel eigenfrequency, this restriction is less critical and thus the high-frequency damping ratio is varied between $0 \leq D_{\mathrm{c}, \text { high }} \leq 0.7$. As can be seen in Fig. 4 (lower), the most comfortable point on the Pareto front in the case of the frequency-selective reference model (Type 1) lies about $15 \%$ beneath the point that results from the standard passive reference model. However, the benefit with regard to ride safety is marginal (about 1\%). When Type 2 of the frequency-dependent reference model is applied, the comfort-optimal setting achieves a weighted chassis acceleration rms value, that is about $25 \%$ lower
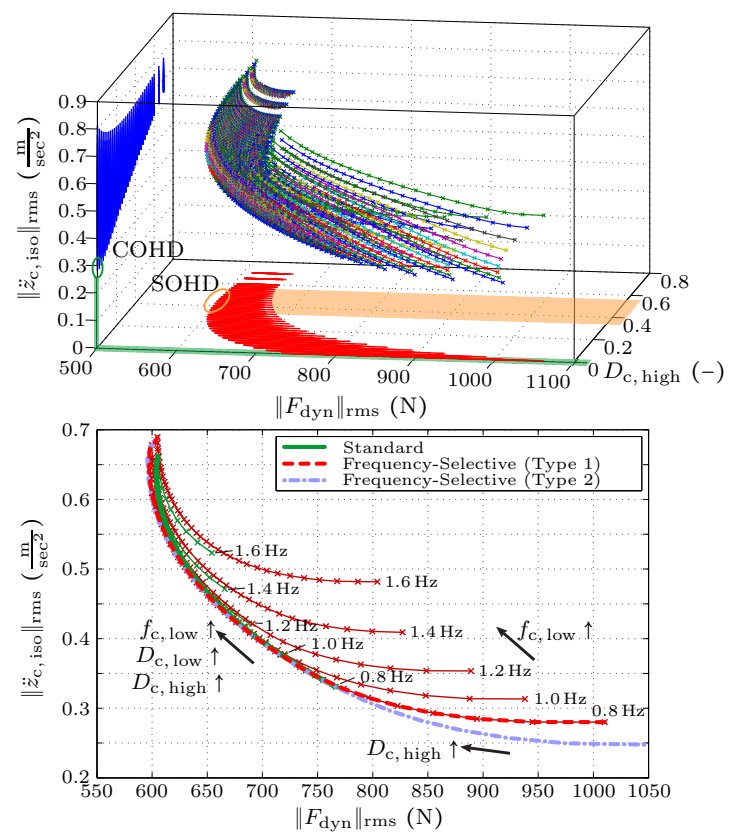

Fig. 4. Conflict diagram for various combinations of $f_{\mathrm{c} \text {, low }}$,

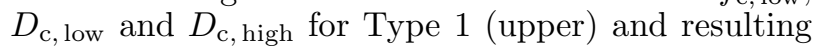
Pareto-optimal front in comparison to the one of the standard passive reference model (lower) 
than in the case of the non-frequency-selective reference, see blue dash-dotted line in Fig. 4 (lower).

The thin lines in Fig. 4 (lower) indicate curves of constant natural frequency $f_{\mathrm{c}} / f_{\mathrm{c} \text {, low }}$ for the frequency-selective and the standard reference model. So, also for semi-active suspension configurations where only damping is varied, the potential for comfort improvement resulting from the new frequency-selective damping approach becomes apparent. However, since the comfort gain in both the active and the semi-active case is at the expense of the road-holding performance the comfort potential may be used by means of adaptation when no safety-critical driving state is determined.

\subsection{Adaptation laws}

Based on the Pareto front discussed above, adaptation laws are derived for use within the suspension control structure. Therefore the adaptation signals $q_{\mathrm{fdyn}}$ and $q_{\mathrm{susp}}$ are mapped to the identified Pareto-optimal front. By doing so, the desired suspension setting is adjusted according to the criticality of the current driving state represented by the two adaptation signals. Just as is the case for the standard reference model-based adaptation laws, see Koch et al. (2011b), a rather soft chassis natural frequency $f_{\mathrm{c}, \text { low }}=0.8 \mathrm{~Hz}$ for the low-frequency range is chosen for the uncritical values of $q_{\mathrm{fdyn}}$ and $q_{\text {susp }}$ close to 0 . With rising dynamic tire load and thus with $q_{\text {fdyn }}$ increasing, at first only the chassis damping ratios $D_{\mathrm{c} \text {, low }}$ and $D_{\mathrm{c} \text {, high }}$ are raised, while $f_{\mathrm{c} \text {, low }}$ is kept at a constant level. Only when values of $q_{\mathrm{fdyn}} \geq 0.8$ are exceeded, the natural frequency of the chassis is also shifted to a firmer setting. The lowfrequency damping ratio already reaches its maximum value of $D_{\text {c, low }}=0.7$ at a chassis eigenfrequency of $f_{\mathrm{c}, \text { low }}=$ $0.9 \mathrm{~Hz}$, while the damping ratio for high-frequent motions still amounts to $D_{\mathrm{c}, \text { high }}=0.42$ at the point of optimal safety with $f_{\mathrm{c} \text {, low }}=1.2 \mathrm{~Hz}$, see also SOHD in Fig. 4 (upper). Hence this setting is applied when the dynamic tire load approaches critical values, i. e. $q_{\mathrm{fdyn}} \rightarrow 2$. In comparison to the standard reference model-based approach, where the safety-optimal configuration results from $f_{\mathrm{c}}=1.5 \mathrm{~Hz}$ and $D_{\text {c }}=0.44$, Koch et al. (2011b), in the case of the frequencyselective reference model a considerably lower natural frequency of the chassis is emulated for $q_{\mathrm{fdyn}} \rightarrow 2$. When the suspension deflection approaches its limits and thus for increasing $q_{\text {susp }}$, minimum values for the natural frequency $f_{\mathrm{c}, \text { low, min }}$ and the damping ratios $D_{\mathrm{c} \text {, low } / \text { high, min }}$ are determined that dominate the quantities identified by $q_{\mathrm{fdyn}}$.

In order to gain some more insight into the dynamics of the different reference models, Fig. 5 depicts the frequency responses of $H_{\dot{z}_{\mathrm{g}} \rightarrow \ddot{z}_{\mathrm{c}}}(j \omega)$ and $H_{\dot{z}_{\mathrm{g}} \rightarrow F_{\mathrm{dyn}}}(j \omega)$ denoting the transfer functions from $\dot{z}_{\mathrm{g}}$ to $\ddot{z}_{\mathrm{c}}$ and $F_{\mathrm{dyn}}$, respectively. The standard reference model without frequency selection as well as the frequency-selective references of Type 1 and Type 2 are represented by means of different colors. Each reference model is analyzed for the comfort-oriented setting, i. e. $q_{\mathrm{fdyn}}=0$ (solid lines), and for their respective safety-oriented configuration, i. e. $q_{\mathrm{fdyn}}=2$ (dashed lines). In each case suspension deflection is uncritical, i. e. $q_{\text {susp }}=0$ holds. The frequency responses of the frequency-selective references have been generated by identifying frequencydependent stiffness and damping coefficients $c_{\mathrm{c}}(j \omega)$ and $d_{\mathrm{c}}(j \omega)$ from $(7),(8)$ and $(11),(12)$, respectively, before calculating the transfer functions in the Laplace domain.
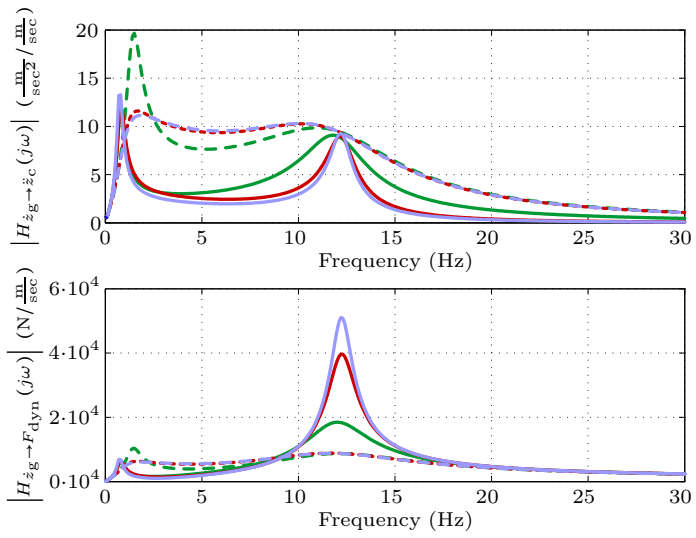

\begin{tabular}{|cccc|}
$\square$ & $q_{\mathrm{fdyn}}=0$ & --- & $q_{\mathrm{fdyn}}=2$ \\
Standard & Type 1 & Type 2 \\
\hline
\end{tabular}

Fig. 5. Frequency response plots of transfer functions $H_{\dot{z}_{\mathrm{g}} \rightarrow \ddot{z}_{\mathrm{c}}}(j \omega)$ (upper) and $H_{\dot{z}_{\mathrm{g}} \rightarrow F_{\mathrm{dyn}}}(j \omega)$ (lower) for $q_{\mathrm{fdyn}}=0$ and $q_{\mathrm{fdyn}}=2$ for different reference models

As can be seen from the upper part of Fig. 5, for $q_{\mathrm{fdyn}}=0$ the frequency-selective reference model is able to improve isolation of the vehicle body from the road disturbance especially in the comfort-relevant frequency range between 4 to $8 \mathrm{~Hz}$. However, in the case of the transfer function related to the dynamic tire load, the peak around the wheel resonance frequency is increased significantly due to the desired damping ratio of $D_{\mathrm{c} \text {, high }}=0$. Hence this setting may only be applied when permitted by the driving conditions. For $q_{\mathrm{fdyn}}=2$, where the frequency-dependent references emulate a lower natural frequency $f_{c \text {, low }}$ and a higher damping ratio $D_{\text {c, low }}$ in the low-frequency range, the peaks in the area of the chassis natural frequency are decreased for both the $\ddot{z}_{\mathrm{c}}$ and the $F_{\mathrm{dyn}}$ transfer function.

\subsection{Control allocation}

Two different force generation devices are provided by the hybrid suspension configuration; an active element, i. e. the spring mount adjustment, is needed for the variation of the chassis natural frequency $f_{\mathrm{c} \text {, low }}$ in the low-frequency range, while the continuously variable damper is used to adjust the damping ratios $D_{\mathrm{c}, \text { low }}$ and $D_{\mathrm{c} \text {, high }}$.

In the case of the frequency-selective reference model of Type 2, the reference actuator forces are determined by

$$
\begin{aligned}
F_{\text {hy }}^{*}(t) & =\left(k_{1} c_{\mathrm{c}, \text { low }}-c_{\mathrm{c}, \text { pass }}(\boldsymbol{x})\right) z_{\mathrm{cw}}, \\
F_{\mathrm{cvd}}^{*}(t) & =d_{\mathrm{c}, \text { high }} \hat{\dot{z}}_{\mathrm{cw}}+k_{2}\left(d_{\mathrm{c}, \text { low }}-d_{\mathrm{c}, \text { high }}\right) \hat{\dot{z}}_{\mathrm{cw}, \text { low }},
\end{aligned}
$$

where $c_{\mathrm{c} \text {, pass }}(\boldsymbol{x})$ incorporates the physical stiffness of the coil spring and the additional buffers that would act between the body and wheel mass if no control action was taken. No lowpass filter is applied to calculate the reference spring force, since the limited bandwidth of the actuator is subsequently accounted for by the real actuator dynamics.

\section{SIMULATION RESULTS}

Simulations are conducted by means of a quarter-car model of the real suspension that incorporates all the nonlinear effects discussed in Section 2 as well as the force generation dynamics of the actuators. A measurement of a rough real country road profile at a vehicle speed of $v_{\mathrm{veh}}=50 \frac{\mathrm{km}}{\mathrm{h}}$ serves as the road excitation. At this speed, the adaptive controller can focus mainly on achieving comfort, since the average values of the adaptation signals $q_{\mathrm{fdyn}}(t)$ 
Table 1. Simulation results for country road profile of bad quality $\left(v_{\mathrm{veh}}=50 \frac{\mathrm{km}}{\mathrm{h}}\right)$

\begin{tabular}{lccccc}
\hline Quantity & & Passive & Standard & Type 1 & Type 2 \\
\hline$\left\|\ddot{z}_{\mathrm{c}}\right\|_{\mathrm{rms}} \quad\left(\frac{\mathrm{m}}{\mathrm{sec}^{2}}\right)$ & 1.71 & 1.24 & 1.30 & 1.19 \\
Benefit vs. passive & - & $27.5 \%$ & $23.8 \%$ & $30.1 \%$ \\
$\left\|\ddot{z}_{\text {c, iso }}\right\|_{\text {rms }} \quad\left(\frac{\mathrm{m}}{\mathrm{sec}^{2}}\right)$ & 1.06 & 0.93 & 0.95 & 0.90 \\
Benefit vs. passive & - & $12.7 \%$ & $10.8 \%$ & $14.9 \%$ \\
$\max \left(\left|\ddot{z}_{\mathrm{c}}\right|\right) \quad\left(\frac{\mathrm{m}}{\mathrm{sec}^{2}}\right)$ & 6.33 & 4.12 & 4.43 & 4.05 \\
Benefit vs. passive & - & $34.9 \%$ & $30.0 \%$ & $36.0 \%$ \\
\hline$\left\|F_{\text {dyn }}\right\|_{\text {rms }} \quad(\mathrm{N})$ & 1158 & 953 & 984 & 953 \\
Benefit vs. passive & - & $17.8 \%$ & $15.0 \%$ & $17.7 \%$ \\
$\min \left(F_{\text {dyn }}\right) \quad(\mathrm{N})$ & -3327 & -2657 & -2820 & -2747 \\
Benefit vs. passive & - & $20.1 \%$ & $15.2 \%$ & $17.4 \%$ \\
$\min \left(z_{\mathrm{cw}}\right) \quad(\mathrm{cm})$ & -8.9 & -6.7 & -6.4 & -6.5 \\
Benefit vs. passive & - & $24.9 \%$ & $28.1 \%$ & $27.0 \%$ \\
\hline$\overline{P^{+}} \quad(\mathrm{W})$ & 0 & 55 & 44 & 62 \\
\hline
\end{tabular}

and $q_{\text {susp }}(t)$ are close to zero. In Table 1, the results of the reference model-based controllers are compared to the performance of the passive configuration. All of the controlled settings are able to improve ride comfort and ride safety over the passive suspension. However, while the frequency-selective reference model of Type 1 - due to the phase distortions in spring and damping forces - cannot enhance the performance of the standard reference either in terms of chassis acceleration or in terms of dynamic tire load, Type 2 of the frequency-selective reference model can. This performance gain, however, comes with an increase in average positive actuator power of $\Delta \overline{P^{+}}=+7 \mathrm{~W}$.

At an increased vehicle speed of $v_{\mathrm{veh}}=75 \frac{\mathrm{km}}{\mathrm{h}}$ on the same road profile the dynamic tire load becomes more critical and the average value of $q_{\mathrm{fdyn}}(t)$ is close to 1 . Hence the reference model-based controller needs to achieve as much comfort as possible while still guaranteeing road-holding properties. The frequency-selective model is able to reduce $\left\|F_{\text {dyn }}\right\|_{\text {rms }}$ by about $2 \%$ in comparison to the standard reference model. While $\left\|\ddot{z}_{\mathrm{c}}\right\|_{\mathrm{rms}}$ can also be improved by about $2 \%,\left\|\ddot{z}_{\mathrm{c} \text {, iso }}\right\|_{\mathrm{rms}}$ deteriorates by about $2 \%$ compared to the standard approach. This is in fact in accordance with Fig. 5, where the standard reference model for higher values of $q_{\mathrm{fdyn}}$ shows superior isolation of the vehicle body from the road unevenness in the comfort-relevant range.

Although the frequency-selective reference model of Type 2 is able to achieve an enhancement over the performance of the standard reference model-based approach, the enormous comfort potential that has been revealed in Fig. 4 cannot be fully exploited by means of the controlled suspension. This is mainly due to the available force range of the continuously variable damper, which is limited by the softest and the hardest damper characteristic illustrated by the solid lines in Fig. 6. The figure also depicts the reference damping forces that were commanded during the simulations with respect to the relative damper velocity for $v_{\mathrm{veh}}=50 \frac{\mathrm{km}}{\mathrm{h}}$ (le.) and $v_{\mathrm{veh}}=75 \frac{\mathrm{km}}{\mathrm{h}}$ (ri.). The damping forces desired by the frequency-selective reference (blue) evidently take up a broader range of values within the force-velocity plane compared to the standard reference (green). At a vehicle speed of $v_{\text {veh }}=50 \frac{\mathrm{km}}{\mathrm{h}}$, this leads to desired forces that only lie within the damper range in about $6 \%$ of the time (compared to $21 \%$ in the case of the standard reference). When the road profile is traveled at a velocity of $v_{\text {veh }}=75 \frac{\mathrm{km}}{\mathrm{h}}$, this number increases to $46 \%$
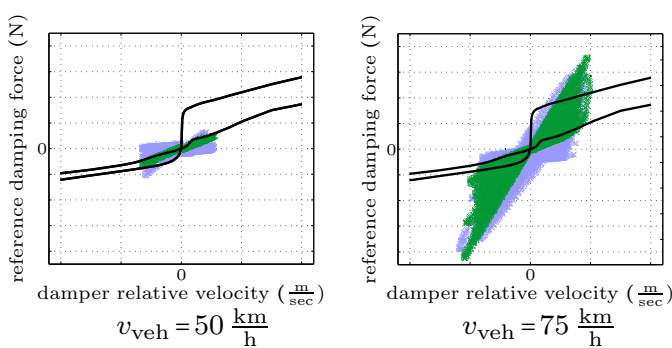

Fig. 6. Desired damping forces vs. limited damper characteristics (green: Standard, blue: Type 2)

of the time interval ( $51 \%$ in the standard case). Since the desired damping forces that lie outside the reachable damper range are clipped to the softest or the hardest characteristic, respectively, this poses a substantial restriction on the possible performance benefit.

\section{CONCLUSION AND OUTLOOK}

In this paper a passive reference model with frequencyselective properties for an adaptive control structure has been derived. Two filtering approaches are designed such that different stiffness and damping coefficients are realized in the low-frequency and the high-frequency range, respectively. Compared to a model without frequency selection, the new reference bares a potential for further comfort improvement of about $25 \%$, particularly within the comfort-relevant frequency range. However, due to the restriction of available damper range, the achievable performance benefit in the application is much smaller.

Future work will investigate how different available damper ranges affect the realizable ride performance. Also, the new frequency-dependent reference model-based control approach needs to be validated using the quarter-car test rig available at the authors' institute.

\section{REFERENCES}

Boivin, D. (2010). Position Sensitive Damper. US Patent 2010/0059321A1.

de Kock, P. and de Ruiter, A.A.W. (2008). Shock Absorber with Frequency-Dependent Damping. US Patent $7395907 \mathrm{~B} 2$.

Hrovat, D. (1997). Survey of advanced suspension developments and related optimal control applications. Automatica, 33(10), 1781 - 1817.

ISO (1997). ISO 2631-1: Mechanical vibration and shockEvaluation of human exposure to whole-body vibration.

Koch, G., Fritsch, O., and Lohmann, B. (2010a). Potential of low bandwidth active suspension control with continuously variable damper. Control Engineering Practice, 18(11), 1251 - 1262

Koch, G., Pellegrini, E., Spirk, S., Pletschen, N., and Lohmann, B. (2011a). Actuator control for a hybrid suspension system. Technical Report 6, No. 2, www.rt.mw.tum.de/en/research/technical-reports/.

Koch, G., Spirk, S., and Lohmann, B. (2010b). Reference model based adaptive control of a hybrid suspension system. In IFAC Symp. Advances in Automotive Control.

Koch, G., Spirk, S., Pellegrini, E., Pletschen, N., and Lohmann, B. (2011b). Experimental validation of a new adaptive control approach for a hybrid suspension system. In 2011 American Control Conference.

Mitschke, M. and Wallentowitz, H. (2004). Dynamik der Kraftfahrzeuge. Springer-Verlag Berlin, 4. edition. 